\title{
Role of the ADCY9 gene in cardiac abnormalities of the Rubinstein-Taybi syndrome
}

\author{
Yueheng $\mathrm{Wu}^{1,2}$, Yu Xia ${ }^{1}$, Ping Li ${ }^{2}$, Hui-Qi Qu ${ }^{3}$, Yichuan Liư ${ }^{3}$, Yongchao Yang ${ }^{1}$, Jijin Lin ${ }^{1}$, Meng Zheng ${ }^{1}$, Lifeng Tian ${ }^{3}$, \\ Zhuanbin Wu', Shufang Huang ${ }^{2}$, Xianyu Qin'1, Xianwu Zhou', Shaoxian Chen', Yanying Liư', Yonghua Wang ${ }^{2}$, \\ Xiaofeng $\mathrm{Li}^{1}$, Hanshi Zeng ${ }^{1}$, Hakon Hakonarson ${ }^{3,5^{*}}$ and Jian Zhuang ${ }^{{ }^{*}}$
}

\begin{abstract}
Background: Rubinstein-Taybi syndrome (RTS) is a rare, congenital, plurimalformative, and neurodevelopmental disorder. Previous studies have reported that large deletions contribute to more severe RTS phenotypes than those caused by CREBBP point mutations, suggesting a concurrent pathogenetic role of flanking genes, typical of contiguous gene syndromes, but the detailed genetics are unclear.

Results: This study presented a rare case of Rubinstein-Taybi (RT) syndrome with serious cardiac abnormalities. Based on the clinical and genetic analysis of the patient, the ADCY9 gene deletion was highlighted as a plausible explanation of cardiac abnormalities. In adcy9 morphant zebrafish, cardiac malformation was observed.

Immunofluorescence study disclosed increased macrophage migration and cardiac apoptosis. RNA sequencing in zebrafish model highlighted the changes of a number of genes, including increased expression of the mmp9 gene which encodes a matrix metalloproteinase with the main function to degrade and remodel extracellular matrix.

Conclusions: In this study, we identified a plausible new candidate gene ADCY9 of CHD through the clinical and genetic analysis of a rare case of Rubinstein-Taybi (RT) syndrome with serious cardiac abnormalities. By functional study of zebrafish, we demonstrated that deletion of adcy 9 is the causation for the cardiac abnormalities. Cardiac apoptosis and increased expression of the MMP9 gene are involved in the pathogenesis.
\end{abstract}

Keywords: $A D C Y 9$, Congenital heart defects, MMP9, Rubinstein-Taybi syndrome

\section{Introduction}

Congenital heart defects (CHDs) are the most common congenital malformations with an incidence of $1 \%$ of live births in developed countries. These are also the leading cause of birth-defect-related deaths [1]. Some CHDs are

\footnotetext{
* Correspondence: Hakonarson@chop.edu; zhuangjiangenetics@163.com ${ }^{3}$ Center for Applied Genomics, The Children's Hospital of Philadelphia, Philadelphia, PA, USA

${ }^{1}$ Guangdong Cardiovascular Institute, Guangdong Provincial Key Laboratory of South China Structural Heart Disease, Guangdong Provincial People's Hospital, Guangdong Academy of Medical Sciences, Guangzhou, Guangdong, China

Full list of author information is available at the end of the article
}

also directly associated with ventricular dysfunction, and heart failure (HF) might be more frequent among pediatric patients with these structural abnormalities [2]. To date, 212 genes have been identified of mutations causing CHDs in human [3], which highlights the complexity of the pathogenesis of CHDs. The identification of CHD causal genes is not only helpful to understand the pathogenesis, but also important in clinical management, such as prenatal molecular diagnosis, management of comorbidities, and selection of the most appropriate surgical interventions.

(c) The Author(s). 2020 Open Access This article is licensed under a Creative Commons Attribution 4.0 International License, which permits use, sharing, adaptation, distribution and reproduction in any medium or format, as long as you give appropriate credit to the original author(s) and the source, provide a link to the Creative Commons licence, and indicate if changes were made. The images or other third party material in this article are included in the article's Creative Commons licence, unless indicated otherwise in a credit line to the material. If material is not included in the article's Creative Commons licence and your intended use is not permitted by statutory regulation or exceeds the permitted use, you will need to obtain permission directly from the copyright holder. To view a copy of this licence, visit http://creativecommons.org/licenses/by/4.0/ The Creative Commons Public Domain Dedication waiver (http://creativecommons.org/publicdomain/zero/1.0/) applies to the data made available in this article, unless otherwise stated in a credit line to the data. 
In the current study, we identified a plausible new candidate gene $A D C Y 9$ of CHD through the clinical and genetic analysis of a rare case of Rubinstein-Taybi (RT) syndrome with serious cardiac abnormalities. By functional study of zebrafish, we demonstrated that deletion of adcy 9 is the causation for the cardiac abnormalities.

\section{Methods}

\section{Clinical case analysis}

The clinical analysis was based on a male neonatal case with cardiac abnormalities admitted to our hospital. The parents and the older sister of the case had normal phenotype. His mother was a 34-year-old female, G4P1Ab2, who had the history of twice induced abortions, once cesarean section, and received obstetric examinations on irregular basis. At 23 weeks of gestation, there was no obvious fetal abnormality shown by the pelvic B-ultrasound results with $3.0 \mathrm{MHz}$ probe. Gestational diabetes of the mother was found at 26 weeks of gestation, with the OGTT results of @fasting 4.71 $\mathrm{mmol} / \mathrm{L}$; @1 h $8.82 \mathrm{mmol} / \mathrm{L} ; @ 2 \mathrm{~h} \quad 6.84 \mathrm{mmol} / \mathrm{L}$. Through diet control, blood glucose of the mother was well controlled. At 27 weeks of gestation, pelvic Bultrasound results revealed fetal heart malformation. The imaging showed that the ascending aorta and aortic arch of the fetus were thin, the aorta was narrowed, and the oval hole was large. Fetal umbilical cord blood was collected at 27 gestational weeks, and fetal chromosome, $\mathrm{Hb}$ electrophoresis, perinatal infection tests (including cytomegalovirus, herpes simplex virus, rubella virus, and Toxoplasma gondii), and chromosomal microarray (CMA) examination were performed on umbilical cord blood. Chromosome exam of fetal umbilical cord blood showed 46, XN, 21pst polymorphism, and the CMA result showed 16p13.3 microdeletion (chr16: 3,721,533-4, 242,948; hg19). The perinatal infection tests were negative. The parents refused to take the CMA examination. A clinical diagnosis of RT syndrome with cardiac abnormalities was made, and the parents chose to continue the pregnancy after informed consent.

Caesarean section was performed at $38+4$ gestational weeks. The infant was delivered with a weight of $3460 \mathrm{~g}$ with Apgar score of 10/10 points. Broad thumbs/toes and short fingers of the infant were observed, while distal joints of the two thumbs broadened more significantly. Muscle strength of the limbs was normal, and no other obvious deformity was found in the outward appearance. On the third day after birth, cardiac ultrasound showed patent ductus arteriosus (PDA), aortic valve dysplastic tricuspid with stenosis, aortic arch dysplasia, minor secondary atrial septal defect, moderate tricuspid regurgitation, severe pulmonary hypertension, and cardiac hypertrophy (asymmetric right ventricular hypertrophy). Annulus diameter of aortic valve cannot be determined ( $Z$ value $=-3.8$ ), with aorta $7.5 \mathrm{~mm}$, diameter of aortic arch $4.2 \mathrm{~mm}(Z$ value $=-4)$, isthmus diameter $4.9 \mathrm{~mm}(Z$ value $=-1)$, distal diameter $8.2 \mathrm{~mm}$ ( $\mathrm{Z}$ value $=1.8)$, arterial catheter $4.4 \mathrm{~mm}$, and bidirectional shunt (online only materials). With the clinical diagnosis of RT syndrome with cardiac abnormalities confirmed, the vital signs and respiratory and circulation status of the patient were closely monitored after birth.

On the third day after birth, the patient developed shortness of breath, increased heart rate and hematochezia. Physical examination showed decreased blood pressure and decreased oxygen saturation, abdominal distension and edema of lower limbs and scrotum. Blood tests revealed decreased platelets and hemoglobin, with carbon dioxide retention. Necrotizing enterocolitis and respiratory failure were diagnosed consequently. Mechanical ventilation, fasting, gastrointestinal decompression, infusion of red blood cells and platelets were administered, and anti-inflammatory treatment was enhanced. However, the general condition of the patient was continuously deteriorating, and the patient left our hospital with his parents at 2 weeks after birth. The final diagnosis at our hospital was: 1 Rubinstein-Taybi syndrome (RTS); 2 congenital heart disease, aortic arch dysplasia, patent ductus arteriosus, atrial septal defect, severe pulmonary hypertension; 3 necrotizing enterocolitis; 4 respiratory failure.

\section{Zebrafish model \\ Zebrafish maintenance}

Adult wild-type $A B$ strain zebrafish were maintained at $28.5^{\circ} \mathrm{C}$ on a $14 \mathrm{~h}$ light $/ 10 \mathrm{~h}$ dark cycle [4]. Five to six pairs of zebrafish were set up for nature mating each time. On average, $200 \sim 300$ embryos were generated. Embryos were maintained at $28.5^{\circ} \mathrm{C}$ in fish water $(0.2 \%$ Instant Ocean Salt in deionized water). The embryos were washed and staged according to standard procedures [5]. The establishment and characterization of the TG (zlyz: EGFP) transgenic lines has been described elsewhere [6]. The zebrafish facility for this study at Shanghai Research Center for Model Organisms is accredited by the Association for Assessment and Accreditation of Laboratory Animal Care (AAALAC) International.

\section{Zebrafish microinjections}

The morpholino (MO) study was designed by Gene Tools, LLC (http://www.gene-tools.com/). Antisense MO was microinjected into fertilized one-cell stage embryos according to standard protocols [7]. The sequence of the adcy 9 exon 3-intron 3 splice-blocking morpholino (adcy9-e3i3-MO) was 5' - TAGTTTGGTTCATCATGT ACCTTGC $-3^{\prime}$, and the sequence for the standard control morpholino was 5'- CСТCTTACCTCAGTTACA ATTTATA - 3' (Gene Tools). For the adcy9 gene 
knock-down experiment, 4 ng of control-MO or adcy9e3i3-MO was used per injection. Total RNA was extracted from 30 to 50 embryos per group in TriPure Isolation Reagent (Roche) according to the manufacturer's instructions. RNA was reverse transcribed using the PrimeScript RT reagent Kit with gDNA Eraser (Takara). Primers spanning adcy9 exon 2 (forward primer: 5TGGGCTCTCAGTGTGGATGTT -3') and exon 4 (reverse primer: 5'- GGAAGGATTGCCGAGTGGT -3') were used for the RT-PCR analysis for confirmation of the efficacy of the adcy9-e3i3-MO (Supplementary Figure 1). The sequences of the primer efl $\alpha$ used as the internal control were 5- GGAAATTCGAGACCAGCA AATAC -3' (forward) and 5'- GATACCAGCCTCAA ACTCACC -3' (reverse).

\section{Acridine orange staining for apoptosis}

Embryos injected with Control-MO and adcy9-e3i3-MO were immersed in $5 \mu \mathrm{g} / \mathrm{ml}$ Acridine orange (AO, acridinium chloride hemi-[zinc chloride], Sigma-Aldrich) in fish water for $60 \mathrm{~min}$ at $3 \mathrm{~d}$ postfertilization (dpf). Next, zebrafish were rinsed thoroughly in fish water for three times ( $5 \mathrm{~min} /$ wash) and anaesthetized with $0.016 \%$ MS222 (tricaine methanesulfonate, Sigma-Aldrich, St. Louis, MO). Zebrafish were then oriented on their lateral side and mounted with $3 \%$ methylcellulose in a depression slide for observation by fluorescence microscopy.

\section{In vivo macrophage migration assays}

To evaluate macrophage migration in zebrafish, fertilized one-cell embryos of TG (zlyz: EGFP) transgenic lines were injected with $4 \mathrm{ng}$ adcy9-e3i3-MO or control-MO. After treatment, all embryos were incubated at $28.5{ }^{\circ} \mathrm{C}$. At $6 \mathrm{dpf}$, embryos were anesthetized with $0.016 \%$ MS222 (tricaine methanesulfonate, Sigma-Aldrich, St. Louis, $\mathrm{MO}$ ) and the number of macrophages recruited to the heart was counted.

\section{Quantitative real-time PCR}

Total RNA was extracted from 30 to 50 embryos per group in Trizol (Roche) according to the manufacturer's instructions. RNA was reverse transcribed using the the PrimeScript RT reagent Kit with gDNA Eraser (Takara). Quantification of gene expression was performed in triplicates using Bio-rad iQ SYBR Green Supermix (Bio-rad) with detection on the Realplex system (Eppendorf). Relative gene expression quantification was based on the comparative threshold cycle method $(2-\Delta \Delta \mathrm{Ct})$ using ef $1 \alpha$ as endogenous control gene. Primer sequences are given in Supplementary Table 1.

\section{Image analysis}

Embryos and larvae were analyzed with Nikon SMZ 1500 Fluorescence microscope and subsequently photographed with digital cameras (5.0 MP). A subset of images was adjusted for levels, brightness, contrast, hue and saturation with Adobe Photoshop 7.0 software (Adobe, San Jose, California) to optimally visualize the expression patterns. Quantitative image analyses were processed using image based morphometric analysis (NIS-Elements D3.1, Japan) and ImageJ software (U.S. National Institutes of Health, Bethesda, MD, USA; http://rsbweb.nih.gov/ij/). Inverted fluorescent images were used for processing. Positive signals were defined by particle number using ImageJ. Ten animals for each treatment were quantified and the total signal per animal was averaged.

\section{RNA sequencing}

In order to better understand the mechanisms of adcy9, we performed RNA sequencing in zebrafish model. Control-MO injected embryos and embryos injected with adcy9-e3i3-MO were frozen at $3 \mathrm{dpf}(1 \mathrm{dpi})$ for RNA-seq analysis. Two biological replicates of $30 \mathrm{em}$ bryos each assay were analyzed in each experimental group. RNA was purified using RNAqueous Total RNA isolation kit (Thermo Fisher). Library preparation and next-generation sequencing were performed at the CCHMC Core Facility using Illumina HiSeq 2500 (75 read length, single sided, $20 \mathrm{M}$ reads per sample). Quality check of the RNA-SEQ reads was performed using Fastqc (http://www.bioinformatics.babraham.ac.uk/projects/fastqc). Reads with low quality adapter content and over-represented sequences were trimmed using SeqPrep (https://github.com/jstjohn/SeqPrep)and Sickle (https://github.com/najoshi/sickle). We mapped and quantified the trimmed RNA-SEQ reads using HISAT2(https://ccb.jhu.edu/software/hisat2/index.shtml) to latest Zebrafish genome assembly GRCz11.92 for each sample at default thresholds.

Differential expression tests of RNA-seq data were performed using the Cuffdiff package in Cufflink2.2.1 [8], and the GTF gene template file was based on $D a$ nio rerio version GRCz11. Library sizes (i.e. sequencing depths) are normalized by the classic-fpkm method. The normality of data distribution was rechecked by visual confirmation. Each protein coding gene was compared the estimated expression level based on measurement of Fragments Per Kilobase of transcript per Million (FPKM). The genes were considered as differentially expressed only if their adjusted $p$ value (q value) less or equal than 0.05 , and the gene enrichment analysis in Gene Ontology (GO, Data Release 2019-01) and biological pathways were done by DAVID v6.8 Bioinformatics platform [9]. A pathway was considered significant by Bonferroni corrected $p<0.05$. 


\section{Statistical analysis}

All data are presented as mean \pm SEM. Statistical analysis and graphical representation of the data were performed using GraphPad Prism 5.0 (GraphPad Software, San Diego, CA). Statistical significance was tested using a Student's $t$ test or $X^{2}$ test as appropriate. Statistical significance is indicated by $*$, where $P<0.05$, and $* \%$, where $P<0.0001$.

\section{Results}

\section{Clinical genetic analysis}

The clinical phenotype of the child includes: 1 broad thumbs/toes; 2 patent foramen ovale, ASD and PDA; 3 aortic development abnormalities (i.e. aortic annulus, ascending aortic coarctation); 4 cardiac hypertrophy; 5 . heart failure; 6 respiratory failure; 7 small intestinal necrosis.

There are several special phenomena worthy of attention in the patient's medical history. First, the ultrasound exam showed normal aortic development at 23 weeks of gestation, but showed that the ascending aorta and aortic arch were slightly thinner at 27 -week, while the aortic annulus and aortic arch were severely constricted after birth. This change indicates that aortic dysplasia in the current case occurred in the late aortic development and deteriorated over time. Secondly, the patient's heart development is basically normal, in which case the aortic malformation is generally surgically treatable. However, after the child was born, he developed rapidly rare complications, including ventricular hypertrophy and heart failure, respiratory failure, and small intestinal necrosis. These suggest a highly complicated nature of the patient's disease, but not a simple cardiac malformation. Thirdly, that the patient's left ventricular development was normal until the child was born. In general, early aortic dysplasia in the fetus (e.g. abnormal aortic dysplasia can be detected by ultrasound at as early as 16 weeks) can lead to left ventricular dysplasia, mainly due to insufficient blood supply [10], but which did not occur in this case. The relatively small effect on left ventricular development in this case might be due to late and less serious aortic dysplasia occurred in the third trimester. Overall, this is a rare and special RTS case, whereas serious complications occurred after birth. Because of these, we decided to investigate its underlying molecular mechanisms through state-of-art technologies and approaches.

\section{Zebrafish model}

The CMA analysis of the patient showed the deletion region of 3,721,533-4,242,948 [hg19] at 16p13.3 and three genes, TRAP1, CREBBP, and $A D C Y 9$, are mapped to this region. Mutations, deletions, and duplications of the $C R E B B P$ gene cause RTS, which is typically characterized by broad thumbs and toes [11]. In animal models, no cardiac abnormalities were recorded [12]. As shown in the literature, large CREBBP deletions contribute to more severe RTS phenotypes than point mutations, implying a concurrent pathogenetic role of flanking genes, a phenomenon typically known as contiguous gene syndromes $[13,14]$. Therefore, it is reasonable to hypothesize that in addition to the CREBBP gene, other genes in the same deleted region may be involved in the disease of the current patient. For the other two genes TRAP1 and $A D C Y 9$, the function of TRAP1 has been relatively clear $[15,16]$. TRAP1 encodes an intra-mitochondrial protein, highly homolog to members of the Hsp90 family, which play fundamental roles in protein folding, protein degradation and signal transduction [17]. Despite these, the Trap1 knockout mice did not have heart abnormalities [18]. Therefore, we proposed $A D C Y 9$ as a plausible candidate to explain the severe heart abnormalities observed in the current patient.

The $A D C Y 9$ gene is conserved across multiple species. Compared with zebrafish, the protein and DNA similarity rates of human $A D C Y 9$ are 65.2 and $64.7 \%$ respectively. To exam the function of $A D C Y 9$ in heart abnormalities, we designed an adcy 9 knockdown experiment in zebrafish via morpholino-modified antisense oligonucleotides. RT-PCR and Sanger sequencing confirmed that the adcy $9 \mathrm{MO}$ experiment was successful, and the target sequence was inserted into the intron 3 of the adcy 9 gene in the MO group.

Heart beat is visible and normal in the control zebrafish, but is abnormal in adcy9-e3i3-MO injected zebrafish (Fig. 1, Supplementary Movie 1, Supplementary Movie 2). Compared with the control zebrafish, the zebrafish with the adcy 9 knockdown has slower heart rate, pericardial edema, small ventricles, cardiac hypertrophy, and atrial blood stagnation. All embryos (100\%) had development defects. The adcy 9 morphant zebrafish has a high mortality rate of $50 \%$ at birth. The cardiac malformation of the adcy 9 morphant zebrafish is mainly presented in the following two aspects: 1 . the ventricle of the adcy 9 morphant zebrafish is smaller than that of the control zebrafish, indicating ventricular dysplasia in the zebrafish; 2 . the adcy9 morphant zebrafish generally have symptoms such as decreased heart rate, pericardial edema, cardiac hypertrophy, and atrial congestion. These indicate that the adcy 9 morphant zebrafish have severe acute heart failure.

\section{Immunofluorescence study on macrophage and apoptosis}

The effect of adcy 9 knockdown on macrophage migration was observed by immunofluorescence technique (Fig. 2). Compared with control fish, embryos injected with adcy9-e3i3-MO showed increased macrophage migration, including the heart. The effect of adcy9 


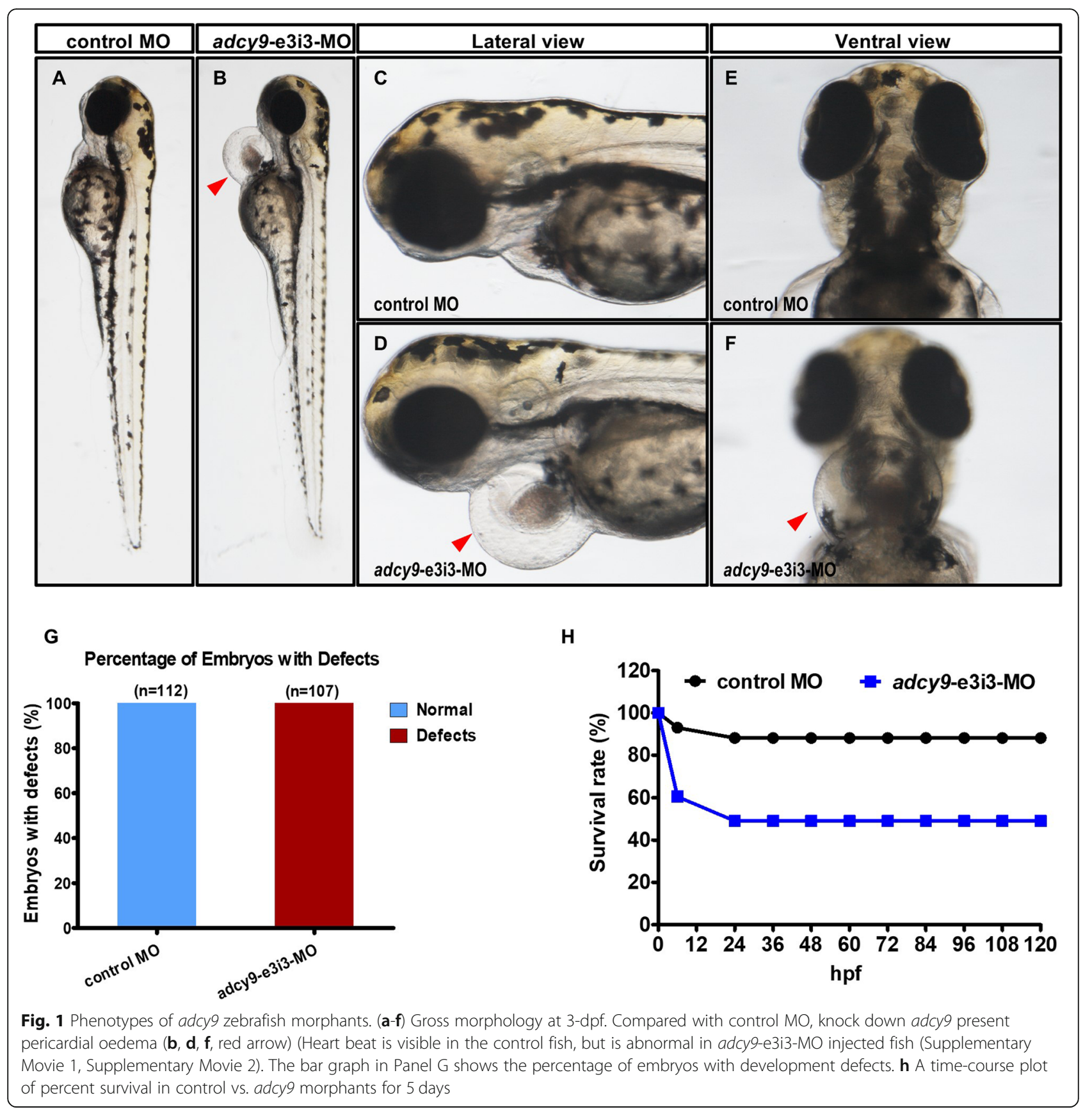

knockdown on zebrafish apoptosis was also observed by immunofluorescence technique. In contrast to control fish, apoptosis was specifically observed in embryonic hearts of adcy9 knockdown zebrafish (Fig. 3). Real-time PCR confirmed that the apoptotic indicator in zebrafish, baxa, had increased expression, while caspases and HDR had reduced expression.

\section{RNA-seq results}

To investigate the molecular mechanisms of acute heart failure and high mortality of adcy9 MO zebrafish, we performed RNA sequencing analysis. The RNA-seq results of differential gene expression analysis comparing zebrafish with or without adcy9 knockdown are shown in Supplementary Table 2. Most significantly, ela2(encoding elastase 2) and cypla (Human ortholog gene CYP1A1, encoding a member of the cytochrome P450 superfamily of enzymes) had decreased expression in the adcy 9 morphant zebrafish. In contrast, these genes had increased expression:

mmp9 (Human ortholog gene MMP9, encoding matrix metallopeptidase 9, involved in the breakdown of extracellular matrix in embryonic development); 


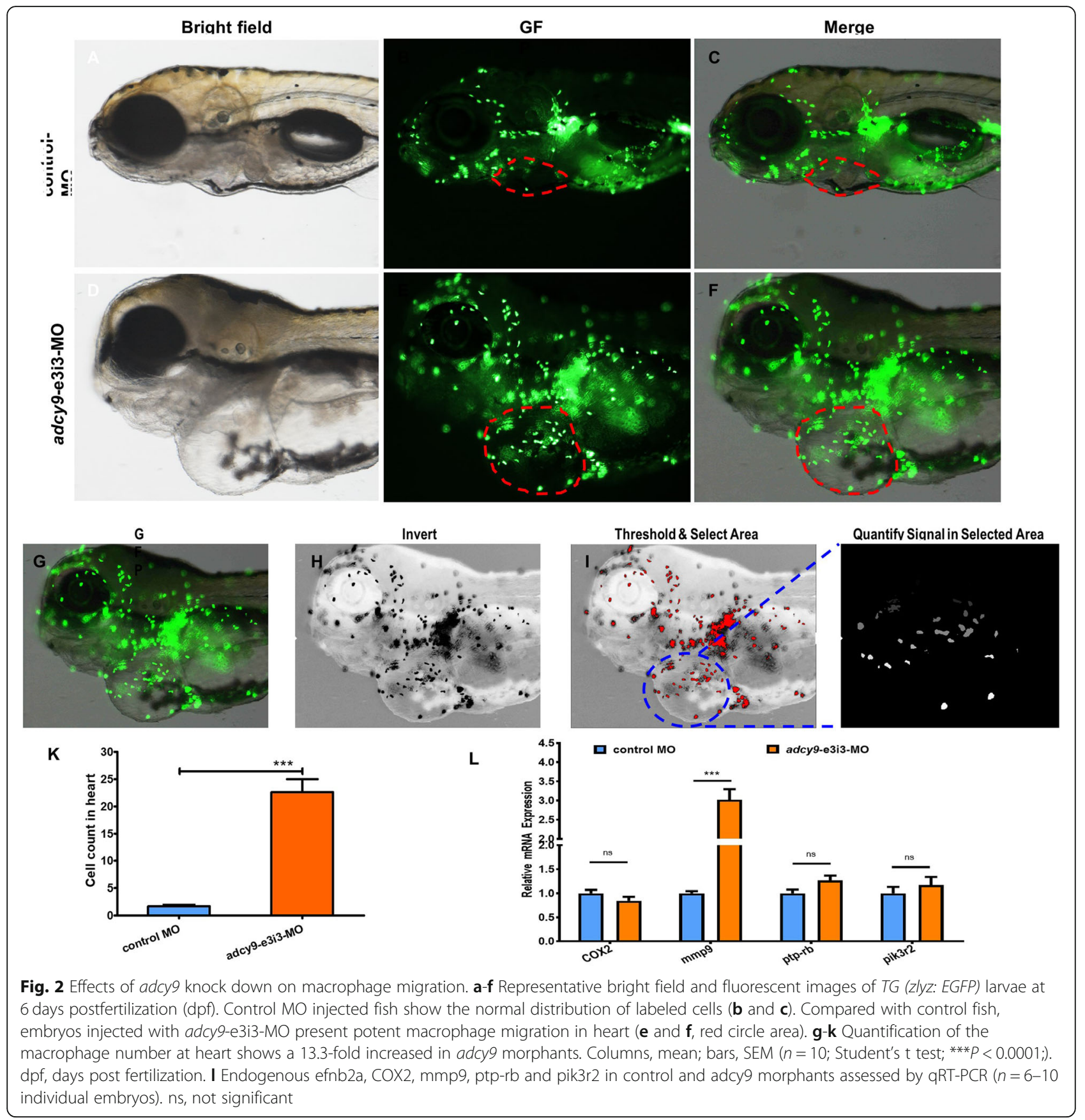

syngap1a (Human ortholog gene SYNGAP1, encoding synaptic Ras GTPase activating protein 1);

fosl1a (Human ortholog gene FOSL1, encoding FOS like 1, AP-1 transcription factor subunit, regulating cell proliferation, differentiation, and transformation);

kank2 (Human ortholog gene KANK2, encoding KN motif and ankyrin repeat domains 2 , involved in cytoskeletal formation by regulating actin polymerization);

acp5a (Human ortholog gene ACP5, encoding acid phosphatase 5 , tartrate resistant); pxnb (Human ortholog gene $P X N$, encoding paxillin, a cytoskeletal protein).

Limited by the sample size of the RNA-seq study, we got 17 genes with $\mathrm{q}$-value $<0.10$, which means $10 \%$ of these genes $(\sim 2$ genes $)$ are false positive. Considering the critical roles of $m m p 9$ in developmental growth and regeneration, we did a realtime PCR experiment and confirmed its higher expression in the morphant zebrafish.

Consequently, we performed GO and Kyoto Encyclopedia of Genes and Genomes (KEGG) analyses 


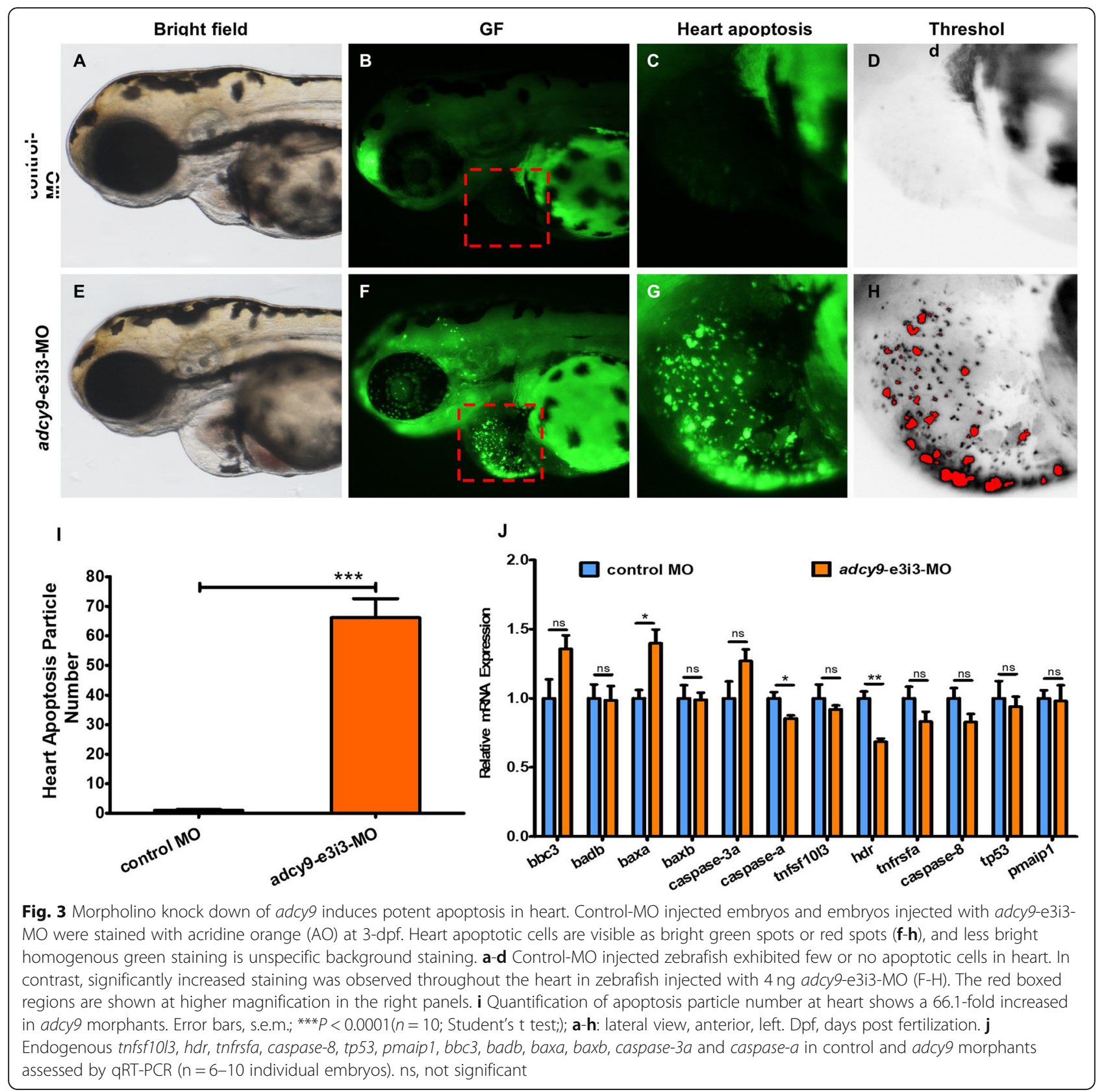

on up-regulated and down-regulated genes (Fig. 4). The GO Biological Processes (BP), the GO Molecular Functions (MF), and the KEGG pathways, highlighted in this study, are shown in Supplementary Table 3. A complete list from the pathway analysis are shown in Supplementary Table 4.

\section{Discussion}

ADCY9: a plausible new candidate gene of CHD

The most common causes of RTS are de novo microdeletions at $16 \mathrm{p} 13.3$, as in this case because the parents have normal phenotype [19]. About 30\% of this syndrome has also cardiac malformations, e.g. patent foramen ovale, ASD, and patent ductus arteriosus [2022]. These heart malformations are usually not serious, and the patients can generally live to adulthood [11, 22]. In contrast, in the current case the phenotype of the patient is more serious and complicated. In addition to the broad thumbs and toes, there were patent foramen ovale, atrial septal defect (ASD), patent ductus arteriosus, aortic developmental defects (aortic annulus, ascending aorta coarctation). Severe phenotypes that are not common in RT syndrome were also seen in this case, including cardiac hypertrophy, left ventricular dysplasia syndrome, heart failure, respiratory failure, and small intestinal necrosis [23, 24]. 

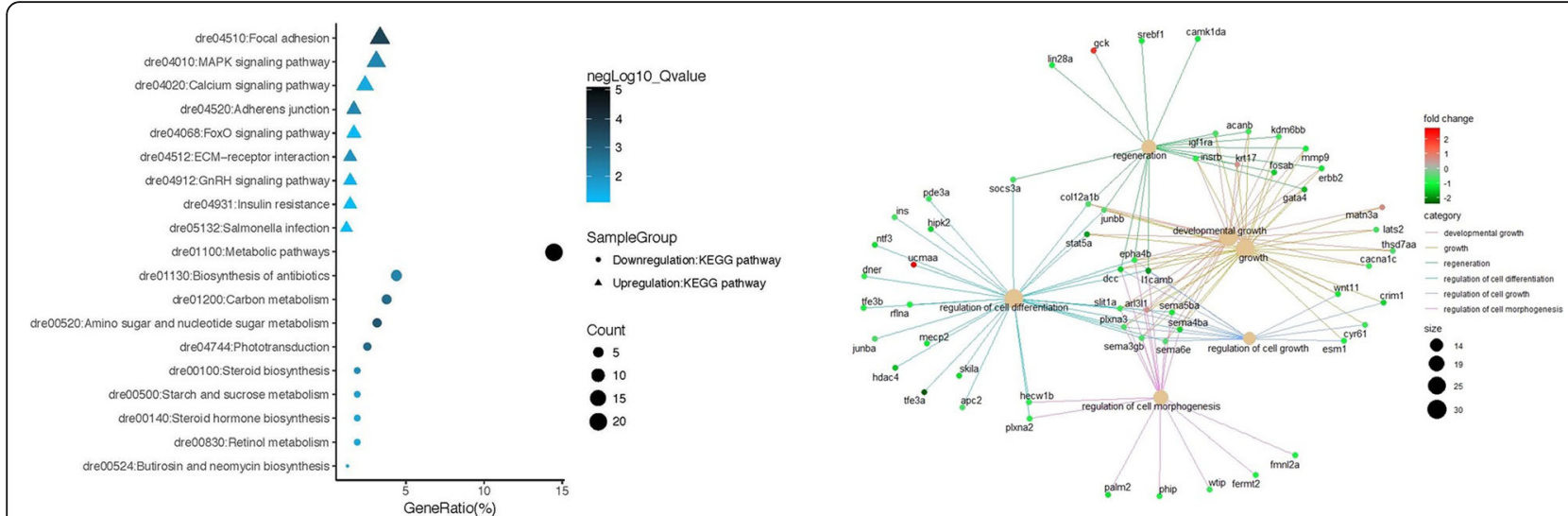

Fig. 4 Scatter plot for KEGG enrichment results. The Gene Ratio is the ratio of differentially expressed gene numbers annotated in this pathway term to all gene numbers annotated in this pathway term. The greater the Gene Ratio, the greater the degree of pathway enrichment. A negLog10_Q value is the corrected $p$ value ranging from 1 to 10, and a higher value indicates greater pathway enrichment

$A D C Y 9$ encodes adenylate cyclase type 9 , an enzyme producing the ubiquitous second messenger cAMP [25]. The ADCY9 gene is composed of five exons and is highly conserved across different species. The previous study by Jin et al. has identified two heterozygous and loss-of-function mutations (LOF, including one splicing mutation and one frame-shifting mutation) in the $A D C Y 9$ gene in a large collection of CHD patients, but no $A D C Y 9$ mutation in controls [3]. Studies have identified the association between single nucleotide polymorphisms (SNP) of ADCY9 (rs1967309) and body mass index (BMI) $[26,27]$. Studies showed also that $A D C Y 9$ polymorphism is associated with cardiovascular disease [28] and cardiovascular outcomes in dyslipidemia treatment [29], but controversies remain [30-32]. Based on the literature and the genetic tests of the current patient, we speculate that the deletion of $A D C Y 9$ gene may be associated with severe heart disease in patients. As shown in our study, adcy 9 morphant zebrafish have very high mortality and deformity rates, indicating the important function of adcy9 in the general and cardiac development of zebrafish.

\section{Myocardial apoptosis in adcy9 morphant zebrafish}

Cardiac hypertrophy was observed in both the patient and adcy 9 morphant zebrafish. Considering the role of myocardial apoptosis in cardiac hypertrophy, we did fluorescence experiments in zebrafish's myocardium and confirmed that myocardial apoptosis occurred in the heart of adcy 9 morphant zebrafish. The signaling pathways of apoptosis in both zebrafish and human can be divided into exogenous and endogenous, while both ultimately lead to high expression of baxa [33, 34]. Our results showed high expression of baxa, which confirmed the occurrence of apoptosis in adcy 9 morphant zebrafish. However, the signaling molecules, caspase-a and
HDR, of exogenous apoptotic signaling pathways were significantly reduced, suggesting that the apoptosis of adcy 9 morphant zebrafish may come from endogenous pathways, instead of exogenous pathways.

\section{mmp9 in cardiac abnormalities in adcy 9 morphant zebrafish}

A number of genes were highlighted in the RNA-seq results to explain the phenotypes of adcy9 MO zebrafish. Among these genes, $m m p 9$ has been demonstrated of critical roles in cardiac development. $m m p 9$ encodes a matrix metalloproteinase whose main function is to degrade and remodel extracellular matrix, and plays critical roles in the formation and function of the placenta and the fetal organs [35]. The $m m p 9$ protein is mainly produced by macrophages [36]. Our results showed that macrophage migration in MO zebrafish was increased, which is concordant with the high expression of mmp9. mmp9 has been found to be associated with numerous pathological processes, including cancer [37], viral infection [38], and blood lipoprotein levels [39]. In addition, mmp 9 is associated with a variety of cardiovascular diseases, e.g. idiopathic atrial fibrillation [40], aortic aneurysms [41] And heart failure [42, 43]. Toba et al. showed that transgenic overexpression of MMP-9 in macrophages exacerbates the effects of MMP-9 in cardiac aging, leading to greater inflammation and fibrosis, which may exacerbate myocyte hypertrophy [44]. MMP9 contributes to cardiovascular remodeling, and $m m p 9$ deletion attenuated thoracic aortic aneurysm formation in mouse model [45]. On the other hand, recent study showed that MMP-9 deficiency augmented angiotensin II-induced abdominal aortic aneurysms [46], related to impair collagen organization and angiogenesis, smooth muscle cell migration and geometrical arterial remodeling [47]. Mmp9 is also associated heart failure [48]. 
Mmp9 deletion may attenuate age-related myocardial fibrosis and diastolic dysfunction [49]. Circulating MMP9 has also been shown to be an effective marker of heart failture and dilated cardiomyopathy [42, 50]. Therefore, higher expression of mmp9 in adcy 9 morphant zebrafish observed in our study may be involved the impairment of extracellular matrix, and cause cardiac abnormalities in zebrafish. There is no known interaction between $A D C Y 9$ and MMP9. The overexpression of mmp9 due to adcy9 knock-down is likely a downstream event with a number of genes in-between.

In conclusion, the case with the 16p13.3 microdeletion syndrome reported in this study had serious aortic dysplasia and heart failure. The $A D C Y 9$ gene in the deleted region is a plausible candidate to explain the cardiac abnormalities. The animal study in adcy 9 morphant zebrafish demonstrated that adcy 9 deletion causes cardiac abnormalities. Increased macrophage migration, cardiac apoptosis, and elevated expression of the $m m p 9$ gene are involved in the pathogenesis. From the clinical aspect, pregnancies with fetal $A D C Y 9$ deletion should be followed closely for aortic dysplasia, even if an initial echocardiogram is normal, to ensure timely surgical correction.

A limitation of this study is that we used the CMA for the genetic testing of the patient. To date, whole exome sequencing (WES) and whole genome sequencing (WGS) have been increasingly used in diagnosing genetic diseases. Compared with WES and WGS, CMA is a well established method, but is relatively old and cannot define the deletion boundary as precise as the former two methods, especially WGS. The zebrafish is a particularly useful model to study gene function in heart development as the embryos can survive without a functional cardiovascular system and blood circulation, by passive diffusion of oxygen [51]. However, we admit that it has limitations. The zebrafish heart has only one atrium and one ventricle, with a primitive outflow tract and without a right ventricle. Therefore, the abnormalities in the patient wouldn't be fully recapitulated by this zebrafish model.

\section{Conclusions}

In this study, we identified a plausible new candidate gene $A D C Y 9$ of CHD through the clinical and genetic analysis of a rare case of Rubinstein-Taybi (RT) syndrome with serious cardiac abnormalities. By functional study of zebrafish, we demonstrated that deletion of adcy9 is the causation for the cardiac abnormalities. Cardiac apoptosis and increased expression of the MMP9 gene are involved in the pathogenesis.

\section{Supplementary information}

Supplementary information accompanies this paper at https://doi.org/10. 1186/s13023-020-01378-9.
Additional file 1. Online only materials

Additional file 2 Supplementary Figure 1. Effectiveness of adcy9 knockdown was confirmed by RT-PCR and sanger sequencing.

Additional file 3 Supplementary Table 1. qRT-PCR primers for Zebrafish

Additional file 4 Supplementary Movie 1. Ventral view.

Additional file $\mathbf{5}$ Supplementary Movie 2. Lateral view.

Additional file $\mathbf{6}$ Supplementary Table 2. Differential gene expression analysis comparing zebrafish with or without adcy9 knockdown

Additional file 7. Supplementary Table 3

Additional file 8. Supplementary Table 4

\section{Abbreviations}

RTS: Rubinstein-Taybi syndrome; RT: Rubinstein-Taybi; CHDs: Congenital heart defects; CHD: Congenital heart disease; CMA: Chromosomal microarray; MO: Morpholino; PDA: Patent ductus arteriosus; KEGG: Kyoto Encyclopedia of Genes and Genomes; GO: Gene Ontology; BMI: Body mass index; ASD: Atrial septal defect; SNP: Single nucleotide polymorphisms

\section{Acknowledgments}

We are grateful to the patients and families for their contributions to this work.

\section{Authors' contributions}

YW: performing the cell experiments, design of the study, analysis and interpretation of data, critical revision of the manuscript; $Y X, P L Y X, S C, H Q$, $Y L, L T$ and $X Q$ : analysis and interpretation of data, drafting manuscript, critical revision of the manuscript content; MZ, $J \mathrm{~L}$ and $\mathrm{ZH}$ : acquisition of clinical data, analysis of data, critical revision of the content; YL: acquisition of clinical data of patients; XL, YW, and ZW: design of the study; $\mathrm{HH}$, and JZ: design of the study, handling funding, writing the paper, full text evaluation and guidance, final approval of the version to be submitted. All authors read and approved the final manuscript.

\section{Funding}

This study was funded by the Guangdong peak project [DFJH201802],the National key Research and Development Program (2018YFC1002600),Science and Technology Planning Project of Guangdong Province, China

(No.2017A070701013,2017B090904034,2017030314109, 2019B020230003).

\section{Availability of data and materials}

All data are fully available without restriction.

\section{Ethics approval and consent to participate}

The experimental protocol was established, according to the ethical guidelines of the Helsinki Declaration and was approved by the Human Ethics Committee of Guangdong Provincial's People Hospital (Institutional Review Board No. GDREC2019354H, Pl: Jian Zhuang). Written informed consent was obtained from individual or guardian participants.

\section{Consent for publication}

Not applicable.

\section{Competing interests}

The authors declare that they have no competing interests.

\footnotetext{
Author details

'Guangdong Cardiovascular Institute, Guangdong Provincial Key Laboratory of South China Structural Heart Disease, Guangdong Provincial People's Hospital, Guangdong Academy of Medical Sciences, Guangzhou, Guangdong, China. ${ }^{2}$ Prenatal Diagnosis Center, Department of Obstetrics and Gynecology, Guangdong Provincial People's Hospital, Guangdong Academy of Medical Sciences, Guangzhou, Guangdong, China. ${ }^{3}$ Center for Applied Genomics, The Children's Hospital of Philadelphia, Philadelphia, PA, USA. ${ }^{4}$ Shanghai Model Organisms Center Inc, Shanghai, China. ${ }^{5}$ Department of Pediatrics and Division of Human Genetics, University of Pennsylvania, Philadelphia, PA, USA.
} 
Received: 17 December 2019 Accepted: 7 April 2020

Published online: 22 April 2020

\section{References}

1. Gilboa SM, et al. Mortality resulting from congenital heart disease among children and adults in the United States, 1999 to 2006. Circulation. 2010; 122(22):2254-63.

2. Hinton RB, Ware SM. Heart failure in pediatric patients with congenital heart disease. Circ Res. 2017;120(6):978-94.

3. Stenson PD, et al. Human gene mutation database (HGMD): 2003 update. Hum Mutat. 2003:21(6):577-81.

4. Westerfield, M., The zebrafish book: a guide for the laboratory use of zebrafish. http://zfin. org/zf_info/zfbook/zfbk. html, 2000.

5. Kimmel CB, et al. Stages of embryonic development of the zebrafish. Dev Dyn. 1995:203(3):253-310.

6. Zhang $Y$, et al. In vivo interstitial migration of primitive macrophages mediated by JNK-matrix metalloproteinase 13 signaling in response to acute injury. J Immunol. 2008;181(3):2155-64.

7. Nasevicius A, Ekker SC. Effective targeted gene 'knockdown'in zebrafish. Nat Genet. 2000;26(2):216.

8. Trapnell C, et al. Differential analysis of gene regulation at transcript resolution with RNA-seq. Nat Biotechnol. 2013;31(1):46-53.

9. Huang DW, et al. DAVID Bioinformatics Resources: expanded annotation database and novel algorithms to better extract biology from large gene lists. Nucleic Acids Res. 2007;35(Web Server issue):W169-75.

10. Hove JR, et al. Intracardiac fluid forces are an essential epigenetic factor for embryonic cardiogenesis. Nature. 2003;421(6919):172-7.

11. Spena S, Gervasini C, Milani D. Ultra-rare syndromes: the example of Rubinstein-Taybi syndrome. J Pediatr Genet. 2015;4(3):177-86.

12. Bartsch $\mathrm{O}$, et al. FISH studies in 45 patients with Rubinstein-Taybi syndrome: deletions associated with polysplenia, hypoplastic left heart and death in infancy. Eur J Hum Genet. 1999;7(7):748-56.

13. Hutchinson DT, Sullivan R. Rubinstein-Taybi Syndrome. J Hand Surg Am. 2015;40(8):1711-2.

14. Stevens CA, Bhakta MG. Cardiac abnormalities in the Rubinstein-Taybi syndrome. Am J Med Genet. 1995;59(3):346-8.

15. Rusconi $D$, et al. Characterization of 14 novel deletions underlying Rubinstein-Taybi syndrome: an update of the CREBBP deletion repertoire. Hum Genet. 2015;134(6):613-26.

16. Hanauer D, Argilla M, Wallerstein R. Rubinstein-Taybi syndrome and hypoplastic left heart. Am J Med Genet. 2002;112(1):109-11.

17. Bartsch $\mathrm{O}$, et al. Evidence for a new contiguous gene syndrome, the chromosome 16p13.3 deletion syndrome alias severe Rubinstein-Taybi syndrome. Hum Genet. 2006;120(2):179-86.

18. Tanaka $Y$, et al. Abnormal skeletal patterning in embryos lacking a single Cbp allele: a partial similarity with Rubinstein-Taybi syndrome. Proc Natl Acad Sci U S A. 1997;94(19):10215-20.

19. Blough Rl, et al. Variation in microdeletions of the cyclic AMP-responsive element-binding protein gene at chromosome band 16p13.3 in the Rubinstein-Taybi syndrome. Am J Med Genet. 2000;90(1):29-34.

20. Stef $M$, et al. Spectrum of CREBBP gene dosage anomalies in RubinsteinTaybi syndrome patients. Eur J Hum Genet. 2007;15(8):843-7.

21. Barbosa, I.A., et al., TRAP1 regulates autophagy in lung cancer cells. Eur J Clin Investig, 2018. 48(4)

22. Li S, et al. Association study of TRAP1 gene polymorphisms with susceptibility and glucocorticoids efficacy of systemic lupus erythematosus. Gene. 2018.

23. Moroni E, Agard DA, Colombo G. The structural asymmetry of mitochondrial Hsp90 (Trap1) determines fine tuning of functional dynamics. J Chem Theory Comput. 2018;14(2):1033-44.

24. Lisanti S, et al. Transgenic expression of the mitochondrial chaperone TNFRassociated protein 1 (TRAP1) accelerates prostate Cancer development. J Biol Chem. 2016;291(48):25247-54.

25. Cumbay MG, Watts VJ. Novel regulatory properties of human type 9 adenylate cyclase. J Pharmacol Exp Ther. 2004;310(1):108-15.

26. Speliotes EK, et al. Association analyses of 249,796 individuals reveal 18 new loci associated with body mass index. Nat Genet. 2010;42(11):937-48.

27. Wen $\mathbf{W}$, et al. Meta-analysis of genome-wide association studies in east Asian-ancestry populations identifies four new loci for body mass index. Hum Mol Genet. 2014;23(20):5492-504.
28. Rautureau Y, et al. ADCY9 (Adenylate Cyclase type 9) inactivation protects from atherosclerosis only in the absence of CETP (Cholesteryl Ester transfer protein). Circulation. 2018;138(16):1677-92.

29. Tardif JC, et al. Pharmacogenomic determinants of the cardiovascular effects of dalcetrapib. Circ Cardiovasc Genet. 2015;8(2):372-82.

30. Pfeffer MA, Dube MP, Tardif JC. Randomized clinical trial needed to confirm whether Dalcetrapib improves outcomes for specific ADCY9 genotype. JAMA Cardiol. 2018;3(9):897.

31. Nissen SE, et al. ADCY9 genetic variants and cardiovascular outcomes with Evacetrapib in patients with high-risk vascular disease: a nested case-control study. JAMA Cardiol. 2018;3(5):401-8.

32. Nissen SE, Pillai SG. Randomized trial needed to confirm whether Dalcetrapib improves outcomes for specific ADCY9 genotype-reply. JAMA Cardiol. 2018;3(9):897-8.

33. Eimon PM, et al. Delineation of the cell-extrinsic apoptosis pathway in the zebrafish. Cell Death Differ. 2006;13(10):1619-30.

34. Eimon PM, Ashkenazi A. The zebrafish as a model organism for the study of apoptosis. Apoptosis. 2010;15(3):331-49.

35. Pustovrh MC, et al. The role of nitric oxide on matrix metalloproteinase 2 (MMP2) and MMP9 in placenta and fetus from diabetic rats. Reproduction 2007;134(4):605-13.

36. Guo G, et al. Induction of macrophage chemotaxis by aortic extracts from patients with Marfan syndrome is related to elastin binding protein. PLoS One. 2011;6(5):e20138.

37. van Kempen LC, Coussens LM. MMP9 potentiates pulmonary metastasis formation. Cancer Cell. 2002;2(4):251-2.

38. Garcia-Etxebarria K, et al. No major host genetic risk factor contributed to a(H1N1)2009 influenza severity. PLoS One. 2015;10(9):e0135983.

39. Kettunen J, et al. Genome-wide study for circulating metabolites identifies 62 loci and reveals novel systemic effects of LPA. Nat Commun. 2016;7:11122.

40. Hsiao FC, et al. MMP9 Rs3918242 polymorphism affects tachycardia-induced MMP9 expression in cultured atrial-derived Myocytes but is not a risk factor for atrial fibrillation among the Taiwanese. Int J Mol Sci. 2016;17(4):521.

41. Ju X, et al. IL-6 regulates extracellular matrix remodeling associated with aortic dilation in a fibrillin-1 hypomorphic mgR/mgR mouse model of severe Marfan syndrome. J Am Heart Assoc. 2014;3(1):e000476.

42. Radosinska J, Barancik M, Vrbjar N. Heart failure and role of circulating MMP2 and MMP-9. Panminerva Med. 2017;59(3):241-53.

43. Moshal KS, et al. Targeted deletion of MMP-9 attenuates myocardial contractile dysfunction in heart failure. Physiol Res. 2008;57(3):379-84.

44. Toba $\mathrm{H}$, et al. Transgenic overexpression of macrophage matrix metalloproteinase-9 exacerbates age-related cardiac hypertrophy, vessel rarefaction, inflammation, and fibrosis. Am J Physiol Heart Circ Physiol. 2017; 312(3):H375-83.

45. Ikonomidis JS, et al. Effects of deletion of the matrix metalloproteinase 9 gene on development of murine thoracic aortic aneurysms. Circulation. 2005;112(9 Suppl):1242-8.

46. Howatt DA, et al. Relaxin and matrix Metalloproteinase-9 in angiotensin IIinduced abdominal aortic aneurysms. Circ J. 2017;81(6):888-90.

47. Galis ZS, et al. Targeted disruption of the matrix metalloproteinase-9 gene impairs smooth muscle cell migration and geometrical arterial remodeling. Circ Res. 2002;91(9):852-9.

48. Moshal KS, et al. Early induction of matrix metalloproteinase-9 transduces signaling in human heart end stage failure. J Cell Mol Med. 2005;9(3):704-13.

49. Chiao YA, et al. Matrix metalloproteinase-9 deletion attenuates myocardial fibrosis and diastolic dysfunction in ageing mice. Cardiovasc Res. 2012;96(3): 444-55.

50. Antonov IB, et al. Matrix Metalloproteinases MMP-1 and MMP-9 and their inhibitor TIMP-1 as markers of dilated cardiomyopathy in patients of different age. Bull Exp Biol Med. 2018;164(4):550-3.

51. Bakkers J. Zebrafish as a model to study cardiac development and human cardiac disease. Cardiovasc Res. 2011;91(2):279-88.

\section{Publisher's Note}

Springer Nature remains neutral with regard to jurisdictional claims in published maps and institutional affiliations. 